\title{
Atomic Resolution Characterization of Pt Based Bi-Metallic Nano-Catalysts Using Aberration Corrected STEM
}

\author{
G. Yang ${ }^{1}, \mathrm{G} . \mathrm{Hu}^{2}, \mathrm{~S}$. Cheng $^{1}, \mathrm{~T}$. Wagberg $^{2}$ \\ ${ }^{1}$ Electronic Materials Research Laboratory, Key Laboratory of The Ministry of Education\& \\ International Center for Dielectric Research, Xi'an Jiaotong University, Xi'an, China \\ ${ }^{2}$ Department of Physics, Umea University, Umea, Sweden
}

The global energy shortage requires the substitute of new energy strategy and various type of new materials are investigated for energy conversion purpose, including thermoelectric, ferroelectric, superconductors, etc.[1,2]. Among them fuel cell batteries are of particular interest due to their high capacity, good stability and reliability. The oxygen reduction reaction (ORR) in the fuel cells plays an important role in the energy generation, but the cost of the cathode/anode catalysts, which mainly contains $\mathrm{Pt}$ or $\mathrm{Pd}$, limit its application due to their high production cost. In order to reduce the cost for the usage of precious materials, abundant materials with relative good catalytic properties, such as Co, $\mathrm{Ni}$, can be synthesized with the Pt or Pd to achieve equal or better catalytic performance than the pure $\mathrm{Pt}$ nano-particle catalysts. In this work, different ratio of $\mathrm{Pt}-\mathrm{Co}, \mathrm{Pt}-\mathrm{Ni}$ and $\mathrm{Pt}-\mathrm{Co}-\mathrm{Cu}$ nanoparticles were synthesized by wet chemical methods. By carefully controlling the experimental parameters, octahedral metal alloys or dodecahedral core shell nanoparticles can be synthesized. The catalytic properties were measured and it was found that some of these nanoparticles have comparable catalytic properties to the commercial $60 \% \mathrm{Pt} /$ Vulcan products.

Scanning transmission electron microscopy (STEM) with energy dispersive x-ray spectroscopy (EDS) and electron energy loss spectroscopy (EELS) were applied to study the microstructure of the catalysts. The atomic resolution $\mathrm{Z}$-contrast imaging (the image contrast is proportional to $\mathrm{Z}^{2}$, where $\mathrm{Z}$ is atomic number) was achieved by JEOL ARM 200F electron microscope with probe spherical aberration corrector. Figure 1 shows the $\mathrm{Z}$-contrast image of the $\mathrm{Pt}_{3} \mathrm{Co}$ dodecahedral nanoparticles. From the image contrast it can be seen that the structure of the particles seems to be core-shell, where the outer bright shell has a distinct boundary with the dark core. Figure 2 shows the $\mathrm{Z}$ contrast image of two nanoparticles with their corresponding EDS mapping result. The distribution of $\mathrm{Pt}$ and Co clearly indicates a core shell structure, while the elemental analysis shows the ratio of Pt:Co is approximately 3:1. As the crystal lattice mismatch between $\mathrm{Co}$ and $\mathrm{Pt}$ is quite large, it is difficult to synthesize the stable bimetallic nanoparticles with pure Co core covered by Pt shells due to the possible strong strain at the Pt-Co interface. Figure 3 shows an EDS linescan result across one core-shell particle, where the thickness of the shell is measured about $1 \mathrm{~nm}$. Different from the reported core-shell structures, where $\mathrm{Pt}$ intensity is normally maximized at the edge and decrease at the center, in these $\mathrm{Pt}_{3} \mathrm{Co}$ particles the $\mathrm{Pt}$ signal reaches its maximum at the center and Co signal has a minimum at the same position. The possible explanation would be the composition of core is the $\mathrm{Pt}$ and Co alloy rather than pure Co cores, and the Co L edge electron energy loss spectra approve the alloy structure by comparing with Co metal and alloy reference spectra [3]. DFT calculation is also used to model the Pt shell structure and explains their good catalytic properties.

The different ratio of Pt:Co would generate bimetallic alloy with different shapes. Same synthesis method was applied to $\mathrm{PtCo}_{1.5}$ and the resulting nanoparticles were found to be octahedral and $\mathrm{Pt}$ was 
found in the edge while Co in the center by EDS. More detailed analysis including EDS mapping, DFT calculation will also be discussed.

\section{References:}

[1] B. Steele et al., Nature, 414 (2001) 345

[2] Z. Peng et al., Science, 337(3012) 56

[3] Zhao et al., J. App. Phys. 108 (2010) 063704

[4] The authors acknowledge the funding from National Natural Science Foundation of China (51202180), the Fundamental Research Funds for the Central Universities in China and the Swedish Research Council (2010-3973)

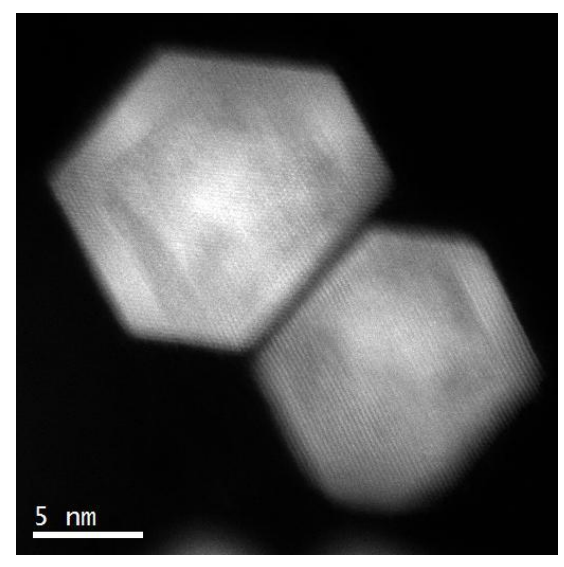

Figure 1. Z-contrast image of $\mathrm{Pt}_{3} \mathrm{Co}$ dodecahedral nanoparticles
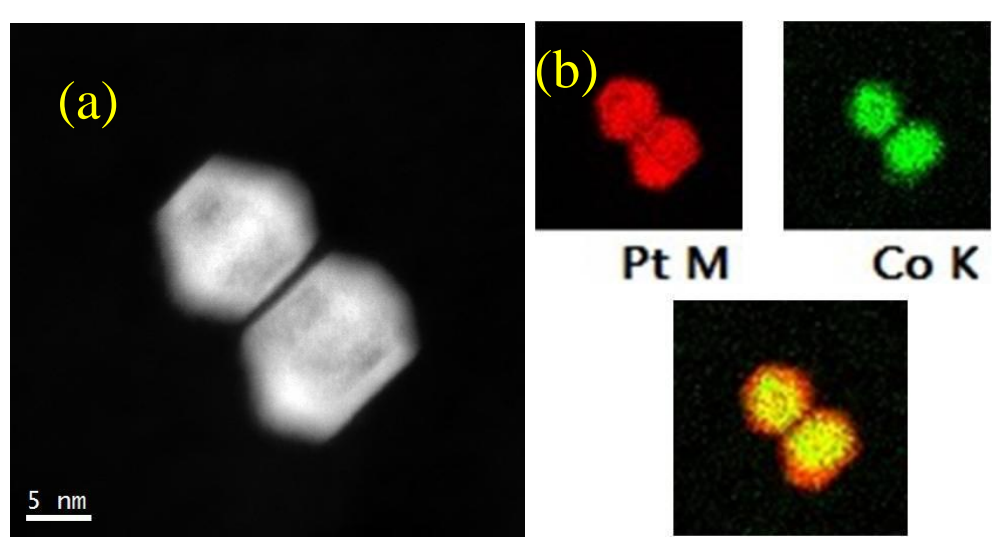

Pt M
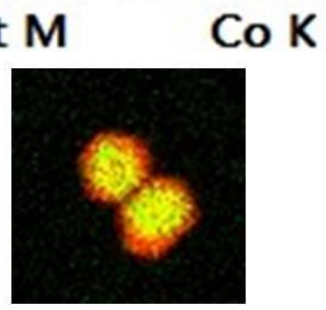

Figure 2. (a) Z-contrast image of $\mathrm{Pt}_{3} \mathrm{Co}$,(b) EDS elemental maps of Pt, Co and a overlapped image

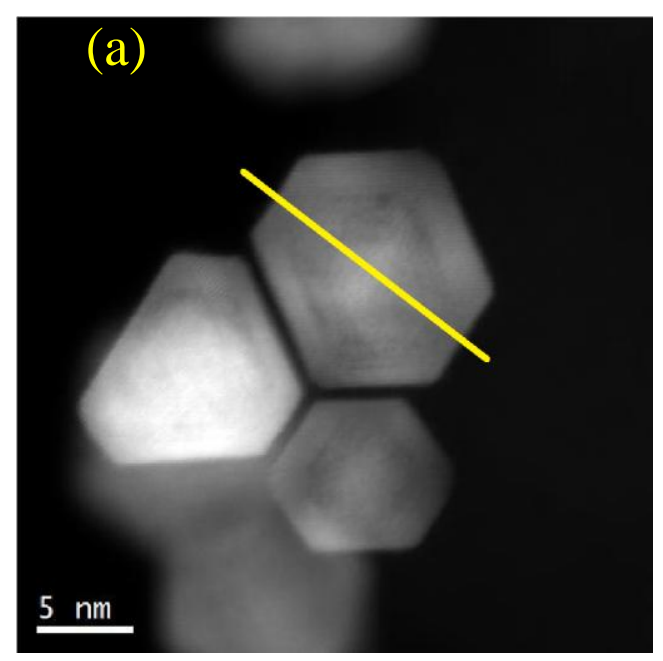

(b)

Figure 3. (a) Z-contrast image of Pt $\mathrm{Cg}$ and the yellow line indicates the EDS linescan position, (b) EDS linescan intensity profiles of Co K peak and Pt M peak. 